\title{
Proton pump inhibitors and the risk of acute kidney injury in older patients: a population-based cohort study
}

Tony Antoniou PhD, Erin M. Macdonald MSc, Simon Hollands MSc, Tara Gomes MHSc, Muhammad M. Mamdani PharmD MPH, Amit X. Garg MD PhD, J. Michael Paterson MSc, David N. Juurlink MD PhD

\section{Abstract}

Background: Proton pump inhibitors (PPIs) cause interstitial nephritis and are an underappreciated cause of acute kidney injury. We examined the risk of acute kidney injury and acute interstitial nephritis in a large population of older patients receiving PPIs.

Methods: We conducted a population-based study involving Ontario residents aged 66 years and older who initiated PPI therapy between Apr. 1, 2002, and Nov. 30, 2011. We used propensity score matching to establish a highly comparable reference group of control patients. The primary outcome was hospital admission with acute kidney injury within 120 days, and a secondary analysis examined acute interstitial nephritis. We used Cox proportional hazards regression to adjust for differences between groups.

Results: We studied 290592 individuals who commenced PPI therapy and an equal number of matched controls. The rates of acute kidney injury (13.49 v. 5.46 per 1000 person-years, respectively; hazard ratio [HR] 2.52, 95\% $\mathrm{Cl} 2.27$ to 2.79 ) and acute interstitial nephritis (0.32 versus 0.11 per 1000 person-years; HR $3.00,95 \% \mathrm{Cl} 1.47$ to 6.14 ) were higher among patients given PPIs than among controls.

Interpretation: In our study population of older adults, those who started PPI therapy had an increased risk of acute kidney injury and acute interstitial nephritis. These are potentially reversible conditions that may not be readily attributed to drug treatment. Clinicians should appreciate the risk of acute interstitial nephritis during treatment with PPIs, monitor patients appropriately and discourage the indiscriminate use of these drugs.

\section{A} cute interstitial nephritis is an immunologically mediated renal injury that is estimated to account for up to one-quarter of cases of acute kidney injury. ${ }^{1,2}$ Of its various causes, idiosyncratic drug reactions are the most important, with antibiotics and nonsteroidal anti-inflammatory drugs (NSAIDs) among the most commonly implicated agents. ${ }^{2}$ Although many patients recover full renal function following discontinuation of the offending drug, some patients transition to chronic kidney disease. ${ }^{1,2}$ Because prompt recognition and withdrawal of precipitant drugs is the cornerstone of managing drug-induced kidney injury, identifying causative medications is essential.

Proton pump inhibitors (PPIs) are among the most widely prescribed medications in the world, with 95 million prescriptions dispensed in the United States in 2009 alone. ${ }^{3}$ Although they are widely perceived as safe, PPIs have been increasingly suspected of causing acute interstitial nephritis, particularly among older patients. ${ }^{4}$ Evidence for this association is limited to anecdotal reports, case series and 3 observational studies, one of which was based on only 5 cases of acute interstitial nephritis and one which examined the risk of acute kidney injury only. ${ }^{4-9}$ Importantly, because classic systemic features of drug-induced acute interstitial nephritis are often absent in PPI-associated cases, the causal role of these drugs may be overlooked.

We sought to compare the risk of acute kidney injury and acute interstitial nephritis in a large population of older patients receiving PPIs with the risk in patients not using these drugs.

Competing interests: During the past 3 years, Muhammad Mamdani has been on advisory boards and/or received honoraria from AstraZeneca, Bristol-Myers Squibb, Eli Lilly and Company, GlaxoSmithKline, Hoffmann-La Roche, Novartis, Novo Nordisk and Pfizer. Amit X. Garg's institution has received an unrestricted educational grant from Pfizer. No other competing interests were declared.

This article has been peer reviewed.

Correspondence to: Tony Antoniou, tantoniou@smh.ca

CMAJ Open 2015. DOI:10.9778/cmajo.20140074 


\section{Methods}

\section{Setting}

We conducted a population-based cohort study involving all residents of Ontario, Canada, aged 66 years and older between Apr. 1, 2002, and Nov. 30, 2011. These individuals had universal access to physician services, hospital care and prescription drug coverage.

\section{Data sources}

We identified prescription drug records using the Ontario Drug Benefit database, which contains comprehensive records of prescription drugs dispensed to all Ontario residents aged 65 years and older. The coding accuracy of information in the database is excellent, with an error rate of less than $1 \% .{ }^{10}$ Because universal coverage for prescription drugs in Ontario begins at the age of 65 years, we restricted our analyses to patients aged 66 years and older to avoid incomplete medication records. We obtained hospital admission data from the Canadian Institute for Health Information's Discharge Abstract Database, which contains detailed clinical information regarding all hospital admissions in Ontario. We used the Ontario Health Insurance Plan database to identify claims for physician services, and the Ontario Diabetes Database to define the presence of diabetes. ${ }^{11}$ We obtained basic demographic data and date of death from the Registered Persons Database, a registry of all Ontario residents eligible for health insurance. These databases were linked in an anonymous fashion using encrypted health card numbers, and are routinely used to explore postmarketing drug safety. ${ }^{12,13}$

\section{Study participants}

We identified 2 groups for comparison. The study group consisted of individuals who commenced treatment with any one of the 5 PPIs available in Ontario during the study period (omeprazole, esomeprazole, lansoprazole, pantoprazole or rabeprazole), and we defined the index date as the date of a patient's first prescription for one of these drugs. Proton pump inhibitors are not available without a prescription in Ontario. To restrict our analysis to patients newly prescribed these drugs, we excluded individuals who received a prescription for any PPI in the 365 days preceding the index date. In both groups, we excluded individuals diagnosed with systemic illnesses associated with interstitial nephritis in the 5 years preceding the index date, including HIV infection, systemic lupus erythematosus and sarcoidosis. ${ }^{1}$ We also excluded individuals with end-stage renal disease (defined as receipt of dialysis in the preceding year or a kidney transplant in the preceding 5 years) and individuals admitted to hospital with acute kidney injury in the year preceding the index date. To avoid the confounding effects of recent illness, we excluded individuals who had been discharged from hospital for any reason in the 30 days preceding the index date. Because acute interstitial nephritis can be provoked by infection or the drugs used to treat it, we excluded individuals diagnosed with any infectious disease as well as those who filled a prescription for an antimicrobial agent in the 120 days preceding cohort entry. Finally, we excluded individuals newly prescribed other medications associated with acute interstitial nephritis, including NSAIDs, loop and thiazide diuretics, anticonvulsants (phenytoin, carbamazepine, phenobarbital, primidone, oxcarbazepine, lamotrigine), histamine $\mathrm{H} 2$ receptor antagonists (ranitidine, cimetidine, famotidine, nizatidine), mesalamine, sulfasalazine, 5-aminosalicylic acid, allopurinol and propylthiouracil. ${ }^{2}$ We defined new use of these drugs as a prescription in the 60 days before cohort entry but no such prescription in the 120 days preceding that prescription. Individuals not satisfying these criteria were considered chronic users of these drugs, and were included in the study.

For each individual prescribed a PPI, we identified one control individual with a randomly assigned index date based on the distribution of index dates in the exposed cohort using propensity score matching. ${ }^{14}$ Variables for the propensity score were selected from among baseline demographic and clinical variables likely to be associated with either a prescription for PPIs or the outcome of acute kidney injury (Appendix 1, available at www.cmajopen.ca/content/1/1/E166/ suppl/DC1). We derived propensity scores for PPI therapy using a nonparsimonious logistic regression model that included PPI exposure as the dependent variable and the baseline variables associated with either the prescription of PPIs or the risk of acute kidney injury. We then used a greedy matching algorithm to pair each patient receiving one of the individual PPIs with an control individual based on the logit of the propensity score (within 0.2 standard deviations), age at index date (within $2 \mathrm{yr}$ ), sex, year of cohort entry, and presence or absence of chronic kidney disease.

\section{Outcomes}

The primary outcome was hospital admission with acute kidney injury (International Statistical Classification of Diseases and Related Health Problems, 10th revision, code N17). We selected this code as our primary outcome rather than the code for acute interstitial nephritis (International Classifications of Diseases, 10th edition, code N10) for several reasons. First, all hospital admissions for acute interstitial nephritis should be encompassed within hospital admissions for acute kidney injury. Second, because acute interstitial nephritis is often undiagnosed as a cause of acute kidney injury, we postulated that the code for acute interstitial nephritis would be an insensitive indicator for drug-related kidney injury. ${ }^{1}$ Finally, the code for acute kidney injury has been validated in our data sets, such that hospital admissions with acute kidney injury represent a median absolute increase in serum creatinine of 98 (interquartile range 43 to 200 ) $\mu \mathrm{mol} / \mathrm{L}$ above the most recent value a median of 39 days preceding hospital admission, whereas absence of the code corresponds to a median absolute increase of 6 (interquartile range -4 to 20) $\mu \mathrm{mol} / \mathrm{L} .{ }^{15}$ Because acute kidney injury is a broad outcome that encapsulates various subtypes of injury, we explored the risk of acute interstitial nephritis in a secondary analysis.

We followed each participant for up to 120 days from their index date until the occurrence of the outcome, death or end 
of the study period (Mar. 31, 2012), whichever occurred first. We selected a 120 day follow-up period because most causes of drug-induced acute interstitial nephritis occur early in the course of therapy. ${ }^{4} \mathrm{We}$ considered only the first outcome in patients with multiple occurrences during the study period. Finally, to test the specificity of our findings with an outcome that should be independent of exposure to PPIs, we examined the association between PPI use and admissions for cataract surgery. Because there is no plausible reason why PPI therapy should be associated with cataract surgery, we reasoned that a null association with this outcome would enhance causal inference in our other analyses.

\section{Statistical analysis}

We computed standardized differences to examine intergroup balance in the distribution of baseline variables. Standardized

\begin{tabular}{|c|c|c|c|c|c|}
\hline \multirow{3}{*}{$\begin{array}{l}\text { Variable } \\
\text { Age, median (IQR), yr }\end{array}$} & \multicolumn{4}{|c|}{ Group; no. $(\%)^{*}$ of patients } & \multirow{3}{*}{$\begin{array}{c}\begin{array}{c}\text { Standardized } \\
\text { difference }\end{array} \\
0.00\end{array}$} \\
\hline & \multicolumn{2}{|c|}{ PPI } & \multicolumn{2}{|c|}{ Control } & \\
\hline & 74 & $(69-80)$ & 74 & $(69-80)$ & \\
\hline $66-74$ & 152738 & $(52.6)$ & 152485 & $(52.5)$ & 0.00 \\
\hline $75-84$ & 103550 & $(35.6)$ & 103628 & $(35.7)$ & 0.00 \\
\hline$\geq 85$ & 34304 & $(11.8)$ & 34479 & $(11.9)$ & 0.00 \\
\hline Female sex & 164724 & $(56.7)$ & 164724 & $(56.7)$ & 0.00 \\
\hline \multicolumn{6}{|l|}{ Charlson Comorbidity Index score } \\
\hline No hospital admission & 104653 & $(36.0)$ & 96921 & (33.4) & 0.06 \\
\hline 0 & 119351 & $(41.1)$ & 128762 & $(44.3)$ & 0.07 \\
\hline 1 & 27954 & $(9.6)$ & 27740 & $(9.5)$ & 0.00 \\
\hline$\geq 2$ & 38634 & $(13.3)$ & 37169 & $(12.8)$ & 0.02 \\
\hline No. of hospital admissions in previous yr & 0 & $(0-0)$ & 0 & $(0-0)$ & 0.02 \\
\hline Residence in a long-term care facility & 13161 & $(4.5)$ & 11889 & $(4.1)$ & 0.02 \\
\hline No. of prescription drugs in previous yr, median (IQR) & 6 & $(3-9)$ & 6 & $(3-9)$ & 0.01 \\
\hline \multicolumn{6}{|l|}{ Medication use in previous $120 \mathrm{~d}$} \\
\hline Oral corticosteroids & 6351 & $(2.2)$ & 5501 & (1.9) & 0.02 \\
\hline Anticoagulants & 16990 & (5.8) & 16662 & (5.7) & 0.01 \\
\hline Calcium channel blockers & 68078 & $(23.4)$ & 69181 & $(23.8)$ & 0.01 \\
\hline $\begin{array}{l}\text { Angiotensin-converting enzyme inhibitor/angiotensin } \\
\text { receptor blocker }\end{array}$ & 110527 & $(38.0)$ & 114843 & $(39.5)$ & 0.03 \\
\hline Potassium-sparing diuretics & 4145 & $(1.4)$ & 3969 & $(1.4)$ & 0.00 \\
\hline Fibric acid derivatives & 5268 & $(1.8)$ & 5522 & $(1.9)$ & 0.01 \\
\hline Metformin & 31198 & $(10.7)$ & 32673 & $(11.2)$ & 0.02 \\
\hline Sulfonylureas & 18753 & $(6.5)$ & 19425 & $(6.7)$ & 0.01 \\
\hline Insulin & 7093 & (2.4) & 7132 & $(2.5)$ & 0.00 \\
\hline Thiazolidinediones & 3290 & $(1.1)$ & 3585 & $(1.2)$ & 0.01 \\
\hline Other oral antihypoglycemics & 1546 & $(0.5)$ & 1500 & $(0.5)$ & 0.00 \\
\hline Chronic NSAIDs & 43719 & $(15.0)$ & 41821 & $(14.4)$ & 0.02 \\
\hline Chronic diuretics & 96659 & (33.3) & 99948 & $(34.4)$ & 0.02 \\
\hline$\beta$-adrenergic antagonists & 65227 & $(22.4)$ & 66329 & $(22.8)$ & 0.01 \\
\hline \multicolumn{6}{|l|}{ Income quintile } \\
\hline 1 (lowest) & 56902 & $(19.6)$ & 55615 & $(19.1)$ & 0.01 \\
\hline 2 & 61151 & $(21.0)$ & 60994 & $(21.0)$ & 0.00 \\
\hline 3 & 57491 & $(19.8)$ & 57942 & $(19.9)$ & 0.00 \\
\hline 4 & 56741 & (19.5) & 57689 & (19.9) & 0.01 \\
\hline 5 (highest) & 57105 & $(19.7)$ & 57463 & $(19.8)$ & 0.00 \\
\hline \multicolumn{6}{|l|}{ Medical conditions and procedures in previous $5 \mathrm{yr}$} \\
\hline Gastrointestinal hemorrhage & 1610 & $(0.6)$ & 1366 & $(0.5)$ & 0.01 \\
\hline Peptic ulcer disease & 25113 & $(8.6)$ & 22756 & $(7.8)$ & 0.03 \\
\hline Esophageal disease & 14557 & $(5.0)$ & 11620 & $(4.0)$ & 0.05 \\
\hline Diabetes & 68921 & $(23.7)$ & 69681 & $(24.0)$ & 0.01 \\
\hline Hypertension & 200516 & $(69.0)$ & 205589 & $(70.7)$ & 0.04 \\
\hline Chronic alcohol use & 7321 & $(2.5)$ & 7371 & $(2.5)$ & 0.00 \\
\hline Systemic malignancy & 13676 & $(4.7)$ & 13486 & (4.6) & 0.00 \\
\hline Chronic kidney disease & 12018 & $(4.1)$ & 12018 & $(4.1)$ & 0.00 \\
\hline Chronic liver disease & 1679 & $(0.6)$ & 1654 & $(0.6)$ & 0.00 \\
\hline Congestive heart failure & 25184 & $(8.7)$ & 22963 & $(7.9)$ & 0.03 \\
\hline
\end{tabular}


differences of less than 0.1 indicate good balance between groups for a given covariate. ${ }^{16} \mathrm{We}$ conducted time-to-event analyses using conditional Cox proportional hazards regression stratified on matched pairs to examine the association of PPIs with acute kidney injury and acute interstitial nephritis, using unexposed individuals as the reference group. We also conducted a stratified analysis of the risk of acute kidney injury for each PPI, to explore whether the risk was consistent across agents. All analyses were conducted using an intentionto-treat approach in which follow-up was not terminated on treatment cessation but was instead only terminated by the occurrence of a primary event or the end of the follow-up interval. We verified the proportional hazards assumption by testing the statistical significance of a time-dependent treatment variable and by visually inspecting the estimated $\log$ ($\log$ ) survival curves. All analyses were performed at the Institute for Clinical Evaluative Sciences (www.ices.on.ca) using SAS version 9.3.

\section{Ethics}

This study was approved by the Research Ethics Board of the Sunnybrook Health Sciences Centre, Toronto.

\section{Results}

During the study period, we identified 290592 individuals who commenced treatment with a PPI and an equal number of matched controls who were highly similar with respect to demographics, medical illnesses and concomitant medications (Table 1). Participants were followed for a median of 120 days, collectively yielding 188869 person-years of follow-up, and the lengths of hospital admissions were 8 and 9 days, respectively. Fourteen patients (all taking PPIs) underwent a renal biopsy during their admission.

In the main analysis, acute kidney injury occurred in 1787 patients within 120 days of the index date. We observed a higher rate of hospital admission with acute kidney injury among patients receiving a PPI than among patients not receiving a PPI (13.49 v. 5.46 per 1000 person-years, respectively; hazard ratio [HR] 2.52, 95\% CI 2.27 to 2.79) (Figure 1,
Table 2). We found similar results in an analysis stratified by individual PPIs (Table 3). We also observed similar results in an analysis stratified by chronic kidney disease. Specifically, the HRs for patients with and without chronic kidney disease were 2.27 (95\% CI 1.82 to 2.84 ) and 2.59 (95\% CI 2.30 to 2.91 ), respectively. In the secondary analysis, we found a similar increase in the risk of acute interstitial nephritis (HR 3.00, 95\% CI 1.47 to 6.14) (Table 2). A total of 48 of 1269 (3.8\%) patients receiving a PPI filled a prescription for an oral corticosteroid in the 14 days following discharge, compared with 9 of 518 (1.7\%) patients who were not prescribed PPIs. As expected, we found no meaningful association between PPI use and cataract surgery (HR 0.97, 95\% CI 0.93 to 1.00 ) (Table 2).

In sensitivity analyses, we found a similar increase in the risk of acute kidney injury among patients receiving PPIs when censoring patients on admission to hospital for infection (HR 2.52, 95\% CI 2.27 to 2.79) or on receipt of other drugs (e.g., antibiotics) classically associated with acute interstitial nephritis (HR 4.03, 95\% CI 3.29 to 4.92). The respective HRs for acute interstitial nephritis were 3.22 (95\% CI 1.53 to 6.81) and 3.25 (95\% CI 1.06 to 9.97) (Appendix 1).

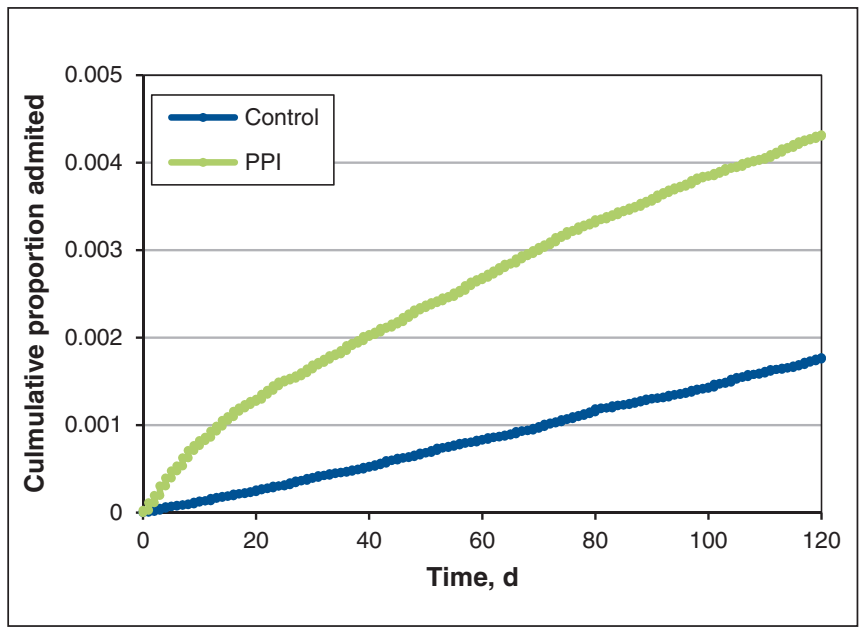

Figure 1: Kaplan-Meier curves for admission to hospital with acute kidney injury, by group.

Table 2: Association between proton pump inhibitor use and kidney outcomes in 290592 patients newly prescribed proton pump inhibitor therapy and an equal number of matched controls

\begin{tabular}{|c|c|c|c|c|c|c|c|}
\hline \multirow[b]{2}{*}{ Variable } & \multicolumn{4}{|c|}{ Group; no. (\%) of events } & \multicolumn{2}{|c|}{ Group; rate per 1000 person-years } & \multirow[b]{2}{*}{$\mathrm{HR}(95 \% \mathrm{Cl})^{*}$} \\
\hline & \multicolumn{2}{|c|}{ PPI } & \multicolumn{2}{|c|}{ Control } & PPI & Control & \\
\hline \multicolumn{8}{|l|}{ Kidney outcomes } \\
\hline Acute kidney injury & 1269 & $(0.4)$ & 518 & $(0.2)$ & 13.49 & 5.46 & 2.52 (2.27 to 2.79$)$ \\
\hline Acute interstitial nephritis & 30 & $(0.0)$ & 10 & $(0.0)$ & 0.32 & 0.11 & $3.00(1.47$ to 6.14$)$ \\
\hline \multicolumn{8}{|l|}{ Tracer outcome } \\
\hline Cataract surgery & 4976 & $(1.7)$ & 5179 & $(1.8)$ & 53.30 & 55.12 & $0.97(0.93$ to 1.00$)$ \\
\hline
\end{tabular}


Because acute kidney injury arising during PPI therapy may not be recognized as interstitial nephritis and therefore not attributed to drug treatment, we speculated that many such patients would resume therapy after discharge. Indeed, of 937 such patients discharged from hospital, more than half $(n=556 ; 59 \%)$ received another prescription for a PPI in the ensuing 100 days. Of these patients 42 (7.5\%) were readmitted to hospital with acute kidney injury in the ensuing 120 days.

\section{Interpretation}

In this population-based study involving nearly 600000 patients, we found that those who commenced treatment with PPIs had a more than twofold increase in the shortterm risk for hospital admission with acute kidney injury relative to patients who were not prescribed these drugs. We observed consistent results in a secondary analysis of admissions with acute interstitial nephritis, and in an analysis stratified by individual PPIs used. Importantly, PPIs were resumed in most patients after discharge from hospital, which highlights the lack of awareness among clinicians of the potential association between these drugs and renal disease. Although only a small proportion of rechallenged patients were subsequently readmitted with acute kidney injury, this finding is consistent with a causal role of PPIs in some instances. Our findings are in general agreement with those of 3 previously reported nested case-control studies, but provide the first estimate of the risk of drug rechallenge among patients admitted with acute kidney injury shortly after initiating PPIs. ${ }^{7-9}$

Our study has important implications for public health. Given the millions of individuals who take PPIs each year and the fact that more than half of such prescriptions may not be clinically indicated, ${ }^{17-20}$ even a small absolute increase in risk of interstitial nephritis outweighs any benefits that might be derived from these drugs in many patients for whom they are prescribed. Although rarely life-threatening, acute interstitial nephritis is a preventable form of iatrogenic harm that may be associated with important sequelae such as hospital admission, dialysis, corticosteroid therapy and chronic kidney disease. ${ }^{1,2}$ Although our study should not deter clinicians from prescrib-

\begin{tabular}{|c|c|c|c|c|}
\hline \multirow[b]{2}{*}{ PPI } & \multirow{2}{*}{$\begin{array}{c}\text { No. } \\
\text { patients } \\
\text { per group }\end{array}$} & \multicolumn{2}{|c|}{$\begin{array}{c}\text { Group; rate per } 1000 \\
\text { person-years }\end{array}$} & \multirow[b]{2}{*}{$\mathrm{HR}(95 \% \mathrm{Cl})^{*}$} \\
\hline & & PPI & Control & \\
\hline Lansoprazole & 27340 & 14.61 & 5.83 & 2.56 (1.85 to 3.55$)$ \\
\hline Omeprazole & 45020 & 12.77 & 4.56 & 2.94 (2.21 to 3.91$)$ \\
\hline Pantoprazole & 57381 & 16.39 & 6.89 & 2.43 (1.97 to 3.00$)$ \\
\hline Rabeprazole & 160851 & 12.48 & 5.14 & 2.45 (2.12 to 2.83$)$ \\
\hline
\end{tabular}

ing PPIs for patients with well-defined indications, our findings underscore the importance of ongoing efforts to curtail the indiscriminate use of these drugs.

\section{Limitations}

Some limitations of our work merit emphasis. We used administrative data and had no access to laboratory indices of renal function, renal biopsy results, treatment indication or medication adherence, and no record of nonprescription medications that may have influenced the risk of acute renal outcomes, including over-the-counter NSAIDs. Our definition of acute kidney injury was broad and undoubtedly included episodes of kidney disease unrelated to PPI use. Similarly, without renal biopsy results, we could not verify the pathology of acute kidney injury. It is therefore likely that we included patients with non-immunologically mediated causes of acute kidney injury in our study, and this may explain the relatively low risk of readmission upon rechallenge with these drugs. However, it is possible that some patients with recurrent disease received treatment as outpatients.

Although we identified admissions for acute kidney injury using a validated algorithm with high specificity (> $95 \%)$, the positive predictive value of the algorithm is decreased for severe disease. The sensitivity of the hospital diagnosis code is limited, particularly for milder forms of the condition in which the incidence of disease can be underestimated up to fivefold compared with definitions using serum creatinine measurements. ${ }^{15}$ In addition, we could not identify mild episodes of acute renal outcomes not culminating in hospital admission. However, this limitation would attenuate the absolute risks we observed. Similarly, the absolute number of patients with acute interstitial nephritis was small, likely reflecting the insensitive nature of codes for this outcome. Although some clinicians appreciate the risk of acute interstitial nephritis with PPIs, differential outcomes ascertainment is unlikely to affect our findings because serum creatinine levels are routinely measured in older patients presenting to hospital. Finally, as with all observational studies, it is possible that our findings partially reflect unmeasured confounders or intergroup differences in the baseline risk of acute renal outcomes.

\section{Conclusion}

We found that older patients who started PPI therapy had an increased risk of acute kidney injury and interstitial nephritis. However, the association between PPIs and acute kidney injury may be overstated given the low risk of recurrence following rechallenge. Clinicians should maintain a high index of suspicion for acute interstitial nephritis among patients taking PPIs who present with a decline in renal function, particularly at the outset of treatment.

\section{References}

1. Praga M. González. Acute interstitial nephritis. Kidney Int 2010;77:956-61.

2. Perazella MA, Markowitz GS. Drug-induced acute interstitial nephritis. Nat Rev Nephrol 2010;6:461-70.

3. Proton pump inhibitors BOCA drug use review and duration of use analysis. Silver Spring (MD): Food and Drug Administration; 2010. Available: www.fda.gov/ 
downloads/advisorycommittees/committeesmeetingmaterials/pediatricadvisory committee/ucm214657.pdf (accessed 2013 Aug. 13).

4. Sierra F, Suarez M, Rey M, et al. Systematic review: proton pump inhibitorassociated acute interstitial nephritis. Aliment Pharmacol Ther 2007;26:545-53.

5. Ray S, Delaney M, Fuller AF. Proton pump inhibitors and acute interstitial nephritis. $B M 7$ 2010;341:c4412.

6. Geevasinga N, Coleman PL, Webster AC, et al. Proton pump inhibitors and acute interstitial nephritis. Clin Gastroenterol Hepatol 2006;4:597-604.

7. Leonard CE, Freeman CP, Newcomb CW, et al. Proton pump inhibitors and traditional nonsteroidal anti-inflammatory drugs and the risk of acute interstitial nephritis and acute kidney injury. Pharmacoepidemiol Drug Saf 2012;21:1155-72.

8. Klepser DG, Collier DS, Cochran GL. Proton pump inhibitors and acute kidney injury: a nested case-control strudy. BMC Nephrol 2013;14:150.

9. Blank ML, Parkin L, Paul C, et al. A nationwide nested case-control study indicates an increased risk of acute interstitial nephritis with proton pump inhibitor use. Kidney Int 2014;86:837-44.

10. Levy AR, O'Brien BJ, Sellors C, et al. Coding accuracy of administrative drug claims in the Ontario Drug Benefit database. Can 7 Clin Pharmacol 2003; 10:67-71.

11. Hux JE, Ivis F, Flintoft V, et al. Diabetes in Ontario: determination of prevalence and incidence using a validated administrative data algorithm. Diabetes Care 2002;25:512-6.

12. Park-Wyllie LY, Juurlink DN, Kopp A, et al. Outpatient gatifloxacin therapy and dysglycemia in older adults. N Engl 7 Med 2006;354:1352-61.

13. Juurlink DN, Mamdani MM, Lee DS, et al. Rates of hyperkalemia after publication of the Randomized Aldactone Evaluation Study. $N$ Engl $7 \mathrm{Med}$ 2004;351:543-51.

14. Austin PC. An introduction to propensity score methods to reduce the effects of confounding in observational studies. Multivariate Behav Res 2011; 46:399-424.

15. Hwang YJ, Shariff SZ, Gandhi S, et al. Validity of the International Classification of Diseases, tenth revision code for acute kidney injury in elderly patients at presentation to the emergency department and at hospital admission. BMF Open 2012;2:e001821.

16. Austin PC, Grootendorst P, Anderson GM. A comparison of the ability of different propensity score models to balance measured variables between treated and untreated subjects: a Monte Carlo study. Stat Med 2007;26:734-53.

17. Forgacs I, Loganayagam A. Overprescribing proton pump inhibitors. BMf 2008;336:2-3.

18. Choudhry MN, Soran H, Ziglam HM. Overuse and inappropriate prescribing of proton pump inhibitors in patients with Clostridium difficile-associated disease. Q7M 2008;101:445-8.

19. Zink DA, Pohlman M, Barnes M, et al. Long-term use of acid suppression started inappropriately during hospitalization. Aliment Pharmacol Ther 2005;21:1203-9
20. Pham CQ, Regal RE, Bostwick TR, et al. Acid suppressive therapy use on an inpatient internal medicine service. Ann Pharmacother 2006;40:1261-6.

Affiliations: Department of Family and Community Medicine (Antoniou), St. Michael's Hospital, Toronto, Ont.; Li Ka Shing Knowledge Institute (Antoniou, Gomes, Mamdani), St. Michael's Hospital, Toronto, Ont.; Institute for Clinical Evaluative Sciences (Antoniou, Macdonald, Hollands, Gomes, Mamdani, Garg, Paterson, Juurlink), Toronto, Ont.; Leslie Dan Faculty of Pharmacy (Gomes, Mamdani), University of Toronto, Toronto, Ont.; Division of Nephrology (Garg), Department of Medicine, Western University, London, Ont.; Department of Epidemiology and Biostatistics (Garg), Western University, London, Ont.; Institute of Health Policy (Paterson), Management and Evaluation, University of Toronto, Toronto, Ont.; Department of Family Medicine (Paterson), McMaster University, Hamilton, Ont.; Departments of Medicine and Pediatrics (Juurlink), University of Toronto, Toronto, Ont.; Sunnybrook Research Institute (Juurlink), Toronto, Ont.

Contributors: All of the authors contributed to the study concept and design, and analysis and interpretation of data. Simon Hollands acquired the data. Tony Antoniou drafted the manuscript, which all of the authors revised. All of the authors gave final approval of the version submitted for publication and agreed to act as guarantors of the work.

Funding: Tony Antoniou is supported by a Canadian Health Institutes of Health Research/Ontario HIV Treatment Network New Investigator Award. This project was supported by research funds from Drug Safety and Effectiveness Research Network and by the Institute for Clinical Evaluative Sciences, which is funded by a grant from the Ontario Ministry of Health and Long-Term Care. The sponsors had no role in the design and conduct of the study; in the collection, analysis, and interpretation of the data; or in the preparation, review, or approval of the manuscript. The opinions, results and conclusions reported in this paper are those of the authors and are independent from the funding sources. No endorsement by the sponsors is intended or should be inferred.

Acknowledgements: The authors thank Brogan Inc., Ottawa, for use of its Drug Product and Therapeutic Class Database; and Hong Zheng for her assistance with data analysis.

Supplemental information: For reviewer comments and the original submission of this article, please see www.cmajopen.ca/content/3/2/E166/supp1/DC1 